\title{
Daniel Schäbler \\ Inszenierungsstrategien einer Ökonomie des Wissens in der Serie CHERnobyL
}

In der 2019 vom US-amerikanischen Streamingdienst HBO produzierten Miniserie CHERNOBYL verkörpert der britische Schauspieler Jared Harris den russischen Chemiker Valeri Legasov (1936-1988). In seiner Küche sitzend, zeichnet Legasov im Prolog der Serie kurz vor seinem Selbstmord folgende Worte auf Tonband auf:

\begin{abstract}
Was ist der Preis von Lügen? Wir verwechseln sie nicht mit der Wahrheit, die wahre Gefahr liegt darin, dass wenn wir genügend Lügen hören, wir die Wahrheit nicht mehr erkennen. [...] Was bleibt anderes übrig, als die Hoffnung auf Wahrheit fallen zu lassen und uns mit Geschichten zu begnügen. In diesen Geschichten geht es nur noch darum, Schuld zuzuschreiben. ${ }^{1}$
\end{abstract}

Legasov, der maßgeblich an der Aufräumaktion nach der Reaktorkatastrophe von Chernobyl 1986 beteiligt war, hat diese Worte vermutlich so nie gesprochen, wohl aber Tonbänder hinterlassen, auf denen er die sowjetische Informationspolitik kritisiert und die Schuld nicht nur bei Einzelnen sieht, sondern im gesamten System verortet. ${ }^{2}$ Indem der Prolog die Opposition zwischen Wahrheit und Lügen eröffnet, lässt diese Eingangsszene bereits erkennen, dass die Serie zentral um das Verhältnis von Fakt und Fiktion kreist, indem sie fragt: Warum und auf welche Weise werden aus tatsächlichen Ereignissen fingierte Ereignisse, mithin Lügen, fabriziert? Zentral ist hierbei auch die Frage nach dem Wissensstand der Beteiligten: Wer hat wann von was gewusst? Wer hat welchen Befehl gegeben? Wie wird die Schuldfrage diskursiv aufbereitet, um die Machtverhältnisse zu bewahren? Authentizität ist damit ein „Effekt““, der „weniger in der Quelle begründet“ ist, sondern vielmehr hervorgerufen wird ,durch die Wirkung bestimmter Vermittlungsstrategien in der Rezeption des Mediennutzers. "3

Den Inszenierungsstrategien, die die Serie zur Verhandlung dieser Fragen wählt, soll im Folgenden nachgegangen und darüber hinaus beleuchtet werden, wie CHeRnobyl auf der Darstellungsebene Authentizität erzeugt, um glaubwür-

1 Chernobyl. Reg. Johan Renck. HBO 2019, TC: 00:00:39-00:01:10.

2 Auch erhängte er sich nicht in seiner Küche, wie in der Serie dargestellt, sondern in seinem Büro. Vgl. Neef, Christian. „,Das Modell taugt nichts““. In: Der Spiegel 12 (2011), 111-112, hier 111. 3 Hattendorf, Manfred. Dokumentarfilm und Authentizität: Ästhetik und Pragmatik einer Gattung. Konstanz: UVK Medien 1999, 67. 
dig zu erscheinen. Es gilt ferner aufzuzeigen, inwiefern die Serie sowohl die Kongruenz und Diskrepanz im (Vor-)Wissen der Figuren in der dargestellten Welt als auch der Rezipient*innen vor dem Bildschirm fruchtbar macht, und daraus eine differenzierte Ökonomie des Wissens erzeugt, die auf Austauschbeziehungen beruht. Mithin soll anhand einer populären Serienproduktion aufgezeigt werden, inwiefern in der Gegenwartskultur Fragen zum Verhältnis zwischen Wahrheit und Lüge, Geschichte und Geschichten durch filmische Authentifizierungsstrategien inszeniert werden.

\section{Genrebestimmung}

Chernobyl lässt sich einer Form der Dokufiktion zuordnen, die als dokumentarisches Historiendrama oder drama documentary bezeichnet worden ist. Markus Wiegandt zufolge handelt es sich dabei um

dramatisierte Dokumentationen (Drama-Documentary), die Ereignisse aus dem realen historischen Geschehen und die Identitäten der beteiligten Protagonisten in ein Filmscript einbinden, eine Form, die ihre Grundlagen im investigativen Journalismus hat und darauf abzielt, Debatten über die verhandelten Ereignisse anzustoßen. ${ }^{4}$

Es handelt sich also um eine auf Recherchen und mithin Quellen basierende Dramatisierung historischer Ereignisse in Spielszenen, die eine zusammenhängende Einheit bilden, also nicht von dominanten Filmdokumenten, Zeitzeugeninterviews oder einer Erzählstimme ergänzt bzw. unterbrochen werden. Dokumentarisch sind diese Filme insofern, als die darin gezeigten Figuren und Schauplätze weitgehend auf historischen Quellen basieren. In den Dialogen zwischen den Figuren erlauben sich diese Produktionen freilich fiktionale Freiheiten und aus Gründen der Dramatisierung gilt dies auch in mehr oder weniger ausgeprägtem Maße für die Ereignisse in der Diegese, auch hier findet man eine Mischung aus Fakt und Fiktion. Es handelt sich also um eine Unterform von Dokufiktion, eine Hybridform, die sich auszeichnet durch ein

Überschreiten der engen Grenzen, in denen sich [...] Dokumentarfilme bewegen. Während der Dokumentarfilm in seiner Intention, dem Einsatz von Effekten und der Wirklichkeitsdarstellung wahrheitsverpflichtet ist, besitzen die Hybridformen gestalterisch wie auch intentional deutlich mehr Freiheiten. ${ }^{5}$

\footnotetext{
4 Wiegandt, Markus. Chronisten der Zwischenwelten: Dokufiktion als Genre. Operationalisierung eines medienwissenschaftlichen Begriffs für die Literaturwissenschaft. Heidelberg: Winter 2017, 45. 5 Wiegandt: Chronisten der Zwischenwelten, 42.
} 
Prominente Markierungen des Dokumentarischen sind etwa eingeblendete Zeitund Ortsangaben, wobei diese oftmals sowohl als genretypisches Signal für Authentizität als auch der Handlungsverortung dienen: „Authentizität ist dabei [...] weniger von der Echtheit als vielmehr von der Glaubwürdigkeit des Dargestellten abhängig. "6

In ihrem Sammelband Echte Geschichte. Authentizitätsfiktionen in populären Geschichtskulturen führen Pirker und Rüdiger folgendes aus: „Medialität manifestiert sich nicht allein im sinnstiftenden Narrativ, also in der Zuschreibung von historischer Bedeutung durch Interpretation und Integration geschichtlicher Daten mit Hilfe von Text, Bild und Ton, sondern auch in der Materialität von Ort und Gegenstand. " 7 Diese Idee und daran anschließende Konzepte der beiden Herausgeber möchte ich im Folgenden um eine rezeptionsästhetische Perspektive erweitern und anhand der Historienserie CHERNOBYL aufzeigen, inwiefern Erinnerungsorte und geschichtliche Ereignisse im kollektiven Gedächtnis einer Rezipient*innengruppe einkodiert sind und in einer Ökonomie des Wissens, die sich zwischen Medium und Publikum entspinnt, eine zentrale Rolle spielen.

\section{Authentizität und Rezeption}

Es handelt sich hierbei wohlgemerkt um ein Versprechen von Authentizität, also einen Pakt mit den Rezipient*innen. ${ }^{8}$ Diese akzeptieren in diesem Rezeptionsmodus die dargestellten Ereignisse als historisch verbürgt, gleichen diese mit ihrem vorhandenen Vorwissen über das Geschehene ab und integrieren das neu hinzugekommene Wissen in ihre Wissensbestände. Dabei lassen sich laut Pirker und Rüdiger zwei dominante Modi innerhalb der Zuschreibungsfelder für das Authentische unterscheiden: zum einen das authentische Zeugnis (Objektauthentizität), zum anderen das authentische Erlebnis (Subjektauthentizität). Medienwissenschaftlich lässt sich diese kleine Typologie mit der Unterscheidung zwischen Makro- und Mikrodrama engführen und präzisieren: Während das Makrodrama die Handlung in historisch-sozialen Umständen situiert und damit auf die Erzeugung von Objektauthentizität abzielt, dreht sich das Mikrodrama um zwischen-

6 Wiegandt: Chronisten der Zwischenwelten, 51.

7 Pirker, Eva Ulrike/Rüdiger, Mark. „Authentizitätsfiktionen in populären Geschichtskulturen: Annäherungen“. In: Echte Geschichte. Authentizitätsfiktionen in populären Geschichtskulturen. Hg. Eva Ulrike Pirker u. a. Bielefeld: transcript 2010, 11-30, hier 18.

8 Pirker/Rüdiger: „Authentizitätsfiktionen in populären Geschichtskulturen“, 14. 
menschliche Konflikte oder Liebesbeziehungen und erzeugt Subjektauthentizität. Pirkers und Rüdigers weiterer Argumentation zufolge

setzen Dokudramen auf detailgenaue Kulissen und glaubwürdige oder plausible Geschichten, um die Zuschauer Geschichte erleben zu lassen, also auf Subjektauthentizität: ,Die Dialoge hätten sich so abspielen können', ist der Grundtenor der Authentizitätsversprechen der Produzenten. Allerdings ist innerhalb dieser vormals klar getrennten Genres eine zunehmende Vermischung von Elementen der Objekt- und Subjektauthentifizierung zu beobachten $[\ldots] .{ }^{9}$

Diese emotionale Aufladung von Fakten in der medialen Aufbereitung speist sich aus einem Verlangen nach authentischen und unverstellten Erfahrungen, mithin etwas, das Walter Benjamin als die „Aura“ eines Kunstwerks bezeichnet hat und das in unserer technisierten und mittlerweile auch digitalisierten Welt ständiger Bedrohung ausgesetzt ist. Es ist dies die „Sehnsucht nach auratischen Erfahrungen [...], die sich besonders deutlich in populären Geschichtskulturen oder auch im Tourismus manifestiert"10. Obgleich zu allen Zeiten oftmals eine Illusion, wird das Auratische trotzdem angestrebt und als etwas Kostbares und unwiederbringlich Verlorenes, oder zumindest akut Bedrohtes figuriert.

\section{Katastrophen inszenieren - das Auratische zwischen Fakt und Fiktion}

Nach diesen Vorüberlegungen möchte ich im Folgenden die Bedeutung des Auratischen für die Figuration einer Ökonomie des Wissens zwischen Medium und Rezipient am Beispiel der aktuell sehr populären dokudramatischen Serie aufzeigen. Zu klären ist hierbei, inwiefern sich die Serie eines mit einer Aura aufgeladenen Erinnerungsortes bedient, um von einem Sog der Faszination im kollektiven Gedächtnis zu profitieren und diesen zu inszenieren.

Die Miniserie CHERnobyl spielt in den heute ukrainischen Ortschaften Chernobyl und Prypiat. Vor April 1986 galt die nur für das neue Kernkraftwerk gebaute Musterstadt Pripyat als Inbegriff der sozialistischen Idee des Fortschritts und des technologisch begründeten Wohlstands. Ab April 1986 wurden die Bilder des evakuierten Ortes ikonisch, standen sie doch für das moralische und tech-

9 Pirker/Rüdiger: „Authentizitätsfiktionen in populären Geschichtskulturen“, 20.

10 Pirker/Rüdiger: „Authentizitätsfiktionen in populären Geschichtskulturen“, 19. 
nologische Scheitern des Sozialismus, der den politischen Zusammenbruch des Ostblocks einleitete, aber auch darüber hinaus für die Gefahren der Atomenergie weltweit. ${ }^{11}$

Albrecht Koschorke zufolge stehen Katastrophen für ein erhöhtes Empfinden des Realen. In der Normalität erscheint das Reale oftmals als „Figur eines katastrophischen Einbruchs“12, als „erkenntnistheoretisches Problem“, das zur „ästhetischen Bearbeitung drängt“, und „,insbesondere mit Begriffen wie Schock oder Trauma“"verbunden ist. ${ }^{13}$ Orte wie Chernobyl und Pripyat fungieren mit Benjamin und Koschorke gesprochen als auratische Erinnerungsorte des Realen, als topographische und symbolische Ankerpunkte im kollektiven Gedächtnis, die besonders anschlussfähig für dokudramatische Inszenierungen erscheinen und die wiederum Authentizitätsfiktionen im Sinne S. J. Schmidts speisen:

\begin{abstract}
Aktanten erzeugen mithilfe von Medien bzw. in Mediensystemen Medientatsachen, und sie erzeugen Authentizitätsfiktionen für ihre Medientatsachen etwa durch Formate und Medienschemata wie TV-Nachrichten oder Reportagen, die eine von Beobachtern unabhängige Fremdreferenz simulieren bzw. diese in der Reaktion von Rezipienten entstehen

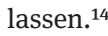

Was Schmidt hier im Rahmen einer allgemeinen Medientheorie formuliert, gilt im Besonderen auch für dokufiktional erzählte Historienstoffe. Serien wie CHERNOBYL funktionieren, weil sie an auratischen Erinnerungsorten spielen sowie eine Reihe von Authentizitätssignalen setzen, wie etwa Einblendungen von Zeit und Ort oder detailgetreue Ausstattung und realistische Effekte. Diesen Gedanken greifen auch Pirker und Rüdiger auf:

11 Das erst mehrere Tage nach der Katastrophe evakuierte und auf viele Generationen hin unbewohnbar gewordene Pripyat, mit seinem weltberühmt gewordenen Riesenrad, ist seitdem als Mahnmal und Erinnerungsort im kollektiven Gedächtnis verankert. Mittlerweile ist es eine Pilgerstätte des sogenannten „schwarzen Tourismus“ geworden, eine gut geölte und profitable Erlebnisindustrie vermarktet diesen Ort soweit, dass inzwischen flächendeckend Mobilfunkmasten aufgestellt werden, damit die Selfies aus den Ruinen rasch und sicher hochgeladen werden können. Vgl. Gerhardt, Daniel: „Der Hype überstrahlt alles“. In: Zeit online vom 19.06.2019. https://www.zeit.de/kultur/film/2019-06/chernobyl-hbo-serie-craigmazin-sky/komplettansicht. (27.06.2020).

12 Koschorke, Albrecht. „Das Mysterium des Realen in der Moderne“. In: Auf die Wirklichkeit zeigen: Zum Problem der Evidenz in den Kulturwissenschaften. Ein Reader. Hg. Helmut Lethen, Ludwig Jäger, Albrecht Koschorke. Frankfurt a. M.: Campus Verlag 2015, 13-38, hier 27.

13 Koschorke: „Das Mysterium des Realen in der Moderne“, 28.

14 Schmidt, Siegfried J. Lernen, Wissen, Kompetenz, Kultur: Vorschläge zur Bestimmung von vier Unbekannten. Heidelberg: Auer 2005, 85. 
Um Authentizitätsfiktionen zu erzeugen, bedarf es einer Annäherung der Authentizitätsvorstellungen von Produzenten und Rezipienten. Je größer die Schnittmenge und die Einbettung in gesellschaftliche Kontexte ist, desto ,authentischer‘ erscheint eine Darstellung. ${ }^{15}$

Hier klingt bereits an, dass ein Austauschprozess von Vor-Wissen vorliegt, bei dem es um die produktionsseitige Antizipation dessen geht, was das Publikum bereits weiß, sowie um die Distribution einer angemessenen - d. h. einer für das Publikum anschlussfähigen und es weder überfordernden noch langweilenden - Menge an Spezialwissen über das historische Ereignis oder den Erinnerungsort. Die Rezipient`innen wiederum gleichen ihr Vorwissen mit der Produktion ab, ergänzen es und integrieren die Authentizitätssignale in den ästhetischen Illusionsprozess. ${ }^{16}$ Aus diesem Prozess ergibt sich ein Schnittpunkt zwischen dem Austauschprozess von (Vor-)Wissen zwischen Produzent`innen und Rezipient`innen und der Authentizitätsfiktion des seriellen historischen Erzählens, der für alle sowohl fiktionalen als auch historisch grundierten Stoffe und Inszenierungen konstitutiv ist.

\section{Das Authentische: Objekt-, Subjekt- und Erlebnisauthentizität}

Nach diesen theoretischen Vorüberlegungen soll im Folgenden die fünfteilige Serie CHERNOBYL konkret in den Fokus genommen werden. Die Serie folgt vier größtenteils historischen Figuren durch die Katastrophe: dem Chemiker Valery Legasov, der Physikerin Ulana Khomyuk, außerdem Lyudmilla Ignatenko, der Frau eines Feuerwehrmanns, und nicht zuletzt Boris Schtscherbina vom Ministerrat der UdSSR. Dabei sind die allermeisten der Figuren historisch verbürgt. Ulana Khomyuk hingegen ist eine fiktionale Mischung verschiedener Personen und verkörpert die aufklärerische Funktion der Wissenschaft, deckt sie doch zusammen mit Legasov die Vertuschung der Katastrophe durch den Staat auf.

Bemerkenswert ist die aufwendige Ausstattung der Serie; so ist etwa die Bildkomposition des Kontrollraums des Reaktors bis ins kleinste Detail, etwa das

15 Pirker/Rüdiger: „Authentizitätsfiktionen in populären Geschichtskulturen“, 21.

16 Zur ästhetischen Illusion vgl. grundlegend Wolf, Werner. Ästhetische Illusion und Illusionsdurchbrechung in der Erzählkunst: Theorie und Geschichte mit Schwerpunkt auf englischem illusionsstörenden Erzählen. Tübingen: Max Niemeyer Verlag 1993. 
Design der Schreibtischlampe, originalgetreu rekonstruiert. ${ }^{17}$ Die gesamte Ausstattung befriedigt sämtliche morbiden Bedürfnisse nach Nostalgie und auch „Ostalgie“. Zum ästhetischen Gesamteindruck passt die Nachbildung von erdiger, robuster, aber heruntergekommener Sowjettechnologie. ${ }^{18}$ Es wurden zudem historische fotografische Dokumente, wie etwa die der Aufräumarbeiten der sogenannten Liquidatoren auf dem Dach des Reaktors, detailgetreu für die Serie rekonstruiert. Zusammen mit unauffällig einmontierten Originaldokumenten, wie etwa den Notfallmeldungen des Kraftwerks an die Feuerwehr oder zeitgenössischen Fernsehbildern und Berichten, wird ein überzeugender Authentizitätseffekt im Sinne Siegfried J. Schmidts generiert, da diese einmontierten Originaldokumente den fremdreferenziellen Gehalt des Erzählten verbürgen und so die Funktion übernehmen, Glaubwürdigkeit zu stiften.

Während Montage und Ausstattung in die Kategorie der „Objektauthentizität“ fallen und damit, freilich mit fiktionalen Freiheiten, ein akkurates Zeugnis repräsentieren, ja den Raum der Katastrophe museal rekonstruieren, verwendet die Serie zusätzlich viel Zeit und Mühe auf die Inszenierungen auf der Ebene der Subjekt- oder Erlebnisauthentizität. Der Fokus liegt hierbei auf kammerspielartig inszenierten Szenen im Reaktorkontrollraum und in anderen Teilen des Kraftwerks, bei denen die Figuren - wie etwa Anatoli Dyatlov, der die Reaktorsteuerung in der Unglücksnacht befehligte - versuchen, mit der Kontingenz der Katastrophe zurechtzukommen. Dyatlov, der durchweg als unsympathischer, zynischer Fanatiker dargestellt wird, weigert sich über lange Zeit, überhaupt die Möglichkeit in Erwägung zu ziehen, dass der Reaktor explodiert sein könnte. Er und Chefingenieur Fomin verweisen reflexartig darauf, dass ein Reaktor vom Typ RBMK bauartbedingt nicht explodieren könne. Den Einschätzungen und Berichten der Mitarbeiter*innen, die den Schaden mit eigenen Augen gesehen haben, werfen sie wahlweise Inkompetenz oder Dummheit vor. Kraft ihres Amtes beanspruchen sie Deutungshoheit über die Ereignisse und stehen damit stellvertretend für unhinterfragbare, politisch etablierte Hierarchien und Machtstrukturen. In der zur

17 So lobt Fountain etwa die „touches of verisimilitude“: „,...] the Unit 4 control room is faithfully re-created, from the control-rod dials on the walls to the white coats and caps worn by the operators“ (Fountain, Henry: „Plenty of Fantasy in HBO’s ,Chernobyl,' but the Truth Is Real“. In: New York Times vom 02.06.2019. https://www.nytimes.com/2019/06/02/arts/television/ chernobyl-hbo.html (27.06.2020)).

18 Im Magazin New Yorker bemerkt hierzu die`der Russland-Expert`in Masha Gessen, „the material culture of the Soviet Union is reproduced with an accuracy that has never before been seen in Western television or film-or, for that matter, in Russian television or film“ (Gessen, Masha: „What HBO’s ,Chernobyl' Got Right, and What It Got Terribly Wrong“. In: The New Yorker vom 04.06.2019. https://www.newyorker.com/news/our-columnists/what-hbos-chernobyl-got-rightand-what-it-got-terribly-wrong (27.06.2020)). 
Schau gestellten Einstellung der politisch verantwortlichen Figuren: „In der Sowjetunion passiert so etwas nicht“, kulminiert die stereotype Darstellung sowjetischer Bunkermentalität, wie sie aus einer westlichen Perspektive oft und gern erzählt wird. In Figurationen wie diesen inszeniert die Serie eine ideologisch aufgeladene Subjektauthentizität, indem sie das spezifisch Totalitäre und Autoritäre als eine eingeschränkte und potenziell desaströse Verarbeitungsstrategie im Angesicht der Katastrophe herausstellt.

In aufwendig animierten Panoramaszenen werden die Verwüstung des Reaktors und das darin lodernde atomare Feuer eindrucksvoll in Szene gesetzt und als eine Form der Objektauthentizität inszeniert. Während das Publikum Vergleichbares nie unmittelbar gesehen hat, wirkt alles ebenso authentisch wie eindrucksvoll hin auf ein ästhetisches Erleben der Katastrophe. Besonders bedrückend sind die Szenen, die zeigen, wie die Feuerwehrmänner ebenso mutig wie ahnungslos in den Reaktor klettern, herumliegendes Graphit aus dem Reaktorkern anfassen und sich somit, auch für das Laienpublikum ersichtlich, einer tödlichen Strahlendosis aussetzen. Bereits hier inszeniert die Serie effektvoll die Diskrepanz zwischen den Wissensbeständen der Figuren und denen des Publikums, das um die tödliche Intensität der Strahlung weiß, während Fomin und Dyatlov in ihrem Bunker arge Probleme haben, funktionsfähige Geigerzähler aufzutreiben, um die Radioaktivität überhaupt zu messen.

Erlebnisauthentizität entspinnt sich auf der Ebene des Mikrodramas, das die schwangere Lyudmila Ignatenko auf der Suche nach ihrem im Einsatz als Feuerwehrmann verstrahlten Mann durch trostlose Krankenhausflure begleitet und damit das menschliche Leid in Szene setzt. In ebenjenem Krankenhaus kommen auch etwas klischeehafte Szenen zwischen trostspendenden Krankenschwestern und versehrten Rettungskräften nicht zu kurz, wobei das Spektakel körperlichen Leids auch intensiv beleuchtet wird, indem die Serie die grauenhaften Folgen der körperlichen Auflösung aufgrund der Strahlenkrankheit in allen Details zeigt. Die Szenen sich plötzlich übergebender Kraftwerksmitarbeiter*innen mit von Strahlung verbrannter Haut erzeugen ein Gefühl des Ekels und der Entfremdung. Größte Bühne für Erlebnisauthentizität ist jedoch der Bereich zwischenmenschlicher Beziehungen. So wird etwa der wachsenden Freundschaft zwischen Legasov und der fiktionalen Komyiuk oder der zunächst sehr schwierigen Beziehung zwischen Legasov und dem anfänglich unnahbaren Politbüro-Funktionär Schtscherbina breiter Raum gegeben. Es menschelt auch unter Kommunist*innen, lautet die wesentliche Erkenntnis. An dieser Inszenierungsstrategie lässt sich die Vermischung von Wissensvermittlung und Dramatisierung exemplarisch beobachten. 


\section{5 Ökonomie des Wissens zwischen Medium und Rezipient*innen}

Die gerade skizzierten Authentizitätseffekte erfüllen zentrale Funktionen innerhalb einer Ökonomie des Wissens. Ein besonderer Fokus liegt dabei auf der Verteilung von Wissen zwischen den Rezipient*innen und der Inszenierung der Diegese. Das ökonomische Prinzip von Angebot und Nachfrage, Überschuss und Mangel, wurde bereits für die Dramentheorie fruchtbar gemacht. In seinem strukturalistischen Standardwerk zum Drama hat Manfred Pfister Ende der 1970er von Informationsvorsprung und Informationsrückstand gesprochen. ${ }^{19}$ Daran anschließend lässt sich zwischen Wissensvorsprung und Wissensrückstand von Figuren und Rezipient^innen unterscheiden. Aus der Diskrepanz zwischen Figuren- und Rezipient`innenwissen ergeben sich vielfältige Möglichkeiten für Einblicke in Machtmechanismen sowie für effektvolle dramatische Ironie. An einigen Beispielen der Serie möchte ich aufzeigen, inwiefern sich Pfisters Typologie, erweitert um von mir entwickelte Kategorien, für die Rolle von dokufiktionaler Authentizität hinsichtlich einer Ökonomie des Wissens fruchtbar machen lässt.

Die durchweg auf Objektauthentizität ausgelegte Ausstattung der Serie weist zunächst einen hohen Grad an Kongruenz zum Vorwissen des Publikums auf. Zugespitzt formuliert: die mise-en-scène erfüllt alle Erwartungen, was das Aussehen der Sowjetunion der 1980er Jahre anbelangt: klobige, robuste, aber aufgrund von Wartungsmängeln auch anfällige Technik, heruntergekommene Wohnräume, farblose Umgebungen und verdreckte Flure. Ob damit Gefühle der Nostalgie oder der Beklemmung aufkommen, liegt an der individuellen Verfasstheit der Rezipient`innen. Unabhängig von einer Wertung durch die Rezipient*innen findet jedoch in jedem Fall durch die inszenierte Nostalgie eine Emotionalisierung des Dargestellten statt, die auf Erinnerung und Wahrnehmung Einfluss nehmen kann. ${ }^{20}$

Eine Inszenierung der Wissensdiskrepanz zwischen Figuren und Rezipient*innen ergibt sich vorrangig aus dem durchgängigen Einsatz von technischem Spezialwissen: Die wenigsten Zuschauer^innen werden mit den Eigenheiten des Reaktortyps „RBMK“ vertraut sein oder wissen, was ein „AZ-5-Schalter“, die

19 Pfister, Manfred. Das Drama. München: Fink 1977, hier 79-89.

$20 \mathrm{Zu}$ Nostalgie und Erinnerung vgl. Gronenthal, Mariella C. Nostalgie und Sozialismus: Emotionale Erinnerung in der deutschen und polnischen Gegenwartsliteratur. Bielefeld: transcript 2018. 
Notfallabschaltung, ist. Insofern herrscht scheinbar zunächst eine Wissensdiskrepanz zwischen Medium und Publikum vor.

Dieses Spezialwissen wird erst in der letzten Folge der Serie im Rahmen des Schauprozesses gegen die Angeklagten Dyatlov und Brukhanov dargeboten. Bis dahin bleibt unklar, wie es genau zur Explosion kam. Durch diese Wissensverteilung ergibt sich ein Spannungsbogen, der erst am Ende der Serie, nach fast fünf Stunden Laufzeit, aufgelöst wird. Das fehlende Spezialwissen und die damit entstehende Asymmetrie zwischen Wissensangebot und Wissensbedarf ist hier also eine wesentliche Inszenierungsstrategie und erzeugt in klassischer Kriminalfilmästhetik Suspense bei den Zuschauern. Der Schauprozess, der mit hohen Haftstrafen für die Verantwortlichen endete, fungiert als eine ideale Bühne für die didaktisch aufbereitete Wissenspräsentation und Wissensverteilung an die Rezipient*innen.

Legasov, als Experte und Zeuge der Anklage, kann mithilfe von Schaubildern schulmeisterlich darlegen, wie der Reaktorkern im Rahmen einer Abschaltübung versehentlich „vergiftet“, also instabil wurde. Als die Leitwarte dann den zu schnell heruntergefahrenen instabilen Reaktor wieder hochfahren wollte, kam es zur Kernschmelze. Auch hier spielt die Absenz von Wissen bzw. vermeintlich unwahrem Wissen eine treibende Rolle: Die Serie zeigt auf, dass die Angeklagten nicht wie jugendliche Raser am Steuer des Kraftwerks saßen, sondern eine komplexe Gemengelage zur Katastrophe führte. Die Kritik hat gerade in Hinblick auf die Wissensdistribution zwischen Figuren und Publikum sensibel reagiert. So bemerkt Nicholson, dass es der Serie gelinge, „to navigate the perilous path of having its characters speak in jargon, and largely refusing to explain it, while keeping viewers on top of what is happening. “21 Hierbei ist gerade die visuelle und nicht so sehr die auditive Ebene der Darstellung hilfreich, da Legasovs Schaubilder die komplexen Zusammenhänge mit nur wenig verbaler Unterstützung auch für Laien verständlich machen. Dieser Informationsrückstand des Publikums ist jedoch insofern unproblematisch und lässt sich leicht $\mathrm{zu}$ einer Kongruenz umformen, da das Publikum das Expertenwissen, zumal es gut nachvollziehbar aufbereitet und erklärt wird, gut in das eigene Vorwissen über Kernenergie integrieren und dadurch verstehen kann, wie es zu Katastrophe kam.

21 Nicholson, Rebecca. „Chernobyl: Horrifying, Masterly Television that Sears on to Your Brain“. In: The Guardian vom 29.05.2019. https://www.theguardian.com/tv-and-radio/2019/may/ 29/chernobyl-horrifying-masterful-television-that-sears-on-to-your-brain (27.06.2020). 


\section{Informationsdiskrepanz}

Der zweite Bereich von Pfisters Typologie, die Informationsdiskrepanz, lässt sich an zwei Beispielen aus der Serie beobachten, aus denen sich ästhetisch und emotional wirkmächtige Szenen ergeben. In mehreren Passagen kurz nach der Katastrophe folgt die Kameraperspektive den Verantwortlichen in der Leitwarte, wenn sie versuchen zu verstehen, was gerade passiert ist: Die Gefühlsschwankungen zwischen Hoffen und Bangen, Beschwichtigungsversuchen und aggressiver Leugnung offensichtlicher Tatsachen kontrastiert mit dem gesicherten Wissen des Publikums, das weiß, dass gerade etwas Schreckliches passiert ist, während die Figuren noch versuchen, dem Störfall im Modus der Routine und Normalität zu begegnen. ${ }^{22}$ Die Figuren wirken schlichtweg von der außergewöhnlichen Situation heillos überfordert und flüchten in fatalen Aktionismus oder ebenso fatale Lethargie. An diesen Stellen wird das Reale besonders greifbar inszeniert, denn es erscheint im Reaktor-Kontrollraum als „Figur eines katastrophischen Einbruchs“"23, als „erkenntnistheoretisches Problem“, das zur „ästhetischen Bearbeitung drängt“. ${ }^{24}$ Die Serie inszeniert dieses epistemologische Problem sowohl ästhetisch als auch narrativ eindrucksvoll als Dilemma für die Figuren, da ihnen unter den gegebenen Umständen wenig Handlungsraum übrigbleibt, sie aber instinktiv starrsinnig handeln und die ihnen vorliegenden Informationen bewusst ignorieren. Das Publikum ist aufgrund seines Wissensvorsprungs den Figuren durchgängig überlegen. Zugleich wird ihm aber auch vor Augen geführt, wie hilflos die unbeherrschbare Nukleartechnik den Menschen zurücklässt.

Eine zweite Szene zeigt die Bevölkerung des nahen Pripyat und inszeniert, wie ganze Familien auf der Straße stehen, dem Feuerschein des explodierten Kraftwerks am Horizont zuschauen und sich am blauen Strahl ionisierter Luft, der senkrecht in den Himmel ragt, erfreuen. ${ }^{25}$ Kinder spielen im radioaktiven

22 Nicht recht nachvollziehbar ist der Eindruck von Hale, der offenbar davon ausgeht, dass das Publikum keinerlei Vorwissen besitzt: „like the operators of the plant, we don't know what has just happened, and we helplessly follow along as they blunder through the flaming wreckage on fruitless tasks [...]“ (Hale, Mike. „,Chernobyl,“ the Disaster Movie“. In: New York Times vom 03.05.2019. https://www.nytimes.com/2019/05/03/arts/television/review-chernobylhbo.html (27.06.2020)). Dieser Eindruck ist insofern überraschend, da es offensichtlich eine Diskrepanz zwischen dem Figuren- und dem Zuschauer*innenwissen geben muss, da die Kraftwerkswarte zunächst davon ausgeht, dass gar kein Unfall stattgefunden hat.

23 Koschorke: „Das Mysterium des Realen in der Moderne“, 27.

24 Koschorke: „Das Mysterium des Realen in der Moderne“, 28.

25 Der effektvoll senkrechte blaue Lichtstrahl wurde in einer Rezension eines Wissenschaftsjournalisten kritisch bewertet: „Don’t get me started about that blue light from the exposed reactor 
Fallout grober Staubpartikel, der wie Schnee herabfällt. Die Szene ist in Zeitlupe inszeniert und wird durch eine extradiegetische Tonspur ergänzt, die langsame Streichermusik einspielt. Die Szene ist damit innerhalb filmischer Konventionen pathetisch, fängt jedoch die Diskrepanz zwischen dem extratextuellen Wissen der Zuschauer*innen und dem Nicht-Wissen der Figuren innerhalb der Diegese effektvoll durch die Inszenierungsstrategien auf extradiegetischer Ebene ein, die als Brücke zwischen diesen beiden Ebenen fungiert. Die Wissensdiskrepanz erzeugt in Kombination mit der Inszenierung eine fast bittere dramatische Ironie und zielt auf Affekte sowohl der Empathie als auch des Entsetzens über die zur Schau gestellte Naivität ab.

\section{Dokufiktion und Kritik}

Ein Seitenblick auf den kritischen Diskurs, den die Serie hervorgerufen hat, beleuchtet schlaglichtartig das in ihr verhandelte und auch von der Kritik als problematisch wahrgenommene Verhältnis zwischen Fakt und Fiktion, gerade in diesem sozio-politisch brisanten Katastrophenfall. Die Reaktionen der Kritiker^innen reichen von großem Zuspruch bis hin zu scharfer Kritik an sachlich oder ethisch fragwürdigen Darstellungsstrategien der Serienmacher. In vielen Fällen geht es genau um das Spannungsfeld zwischen Realitätsnähe und künstlerisch-gestalterischer Freiheit, das für das gesamte Genre der Dokufiktion konstitutiv ist. Anhand zweier Rezensionen der Serie lässt sich die Spannbreite möglicher Reaktionen auf dieses Spannungsverhältnis ablesen.

So räumt etwa Fountain in der New York Times der Serie großzügige fiktionale Freiheiten ein: „The first thing to understand about the HBO mini-series ,Chernobyl $^{`}[. .$.$] is that a lot of it is made up. But here's the second, and more important,$ thing: It doesn't really matter." 26 Dabei fokussiert Fountain vor allem Ungenauigkeiten in der Präsentation der Aufräumarbeiten. So bemerkt er zurecht, dass die Serie den Eindruck erwecke, es seien lediglich die drei Protagonisten für die gesamte Operation verantwortlich, was natürlich aus Gründen der erzählerischen Ökonomie geschieht.

\footnotetext{
shining high into the night sky in the first episode. Yes, nuclear reactors can produce a blue hue, from something called Cherenkov radiation, but no, there's no way Unit 4 would have looked like the ,Tribute in Light“ in Lower Manhattan on the anniversary of Sept. 11.“ (Fountain: „Plenty of Fantasy in HBO’s ,Chernobyl, ‘ but the Truth Is Real“) In diesem Kommentar wird die Spannung zwischen realitätsgetreuer Darstellung und dem Drang nach dramatisch-visuellen Spezialeffekten exemplarisch.

26 Fountain: „Plenty of Fantasy in HBO’s ,Chernobyl,“ but the Truth Is Real“.
} 
Im Kontrast dazu argumentiert Gessen im New Yorker aus einer ethischen Perspektive und moniert die bis ins Verlogene reichende faktische Ungenauigkeit, die die Schuldfrage ideologisch verzerre: „The viewer is invited to fantasize that, if not for Dyatlov, the better men would have done the right thing and the fatal flaw in the reactor, and the system itself, might have remained latent. This is a lie. “27 Gessen präzisiert den Vorwurf und weitet ihn von dieser konkreten Frage auf den gesamten Handlungsaufbau der Serie aus:

Resignation was the defining condition of Soviet life. But resignation is a depressing and untelegenic spectacle. So the creators of "Chernobyl“ imagine confrontation where confrontation was unthinkable - and, in doing so, they cross the line from conjuring a fiction to creating a lie. ${ }^{28}$

Gerade an diesem Zitat lässt sich das Dilemma erkennen, das sich aus der Spannung zwischen Fakt und Fiktion und dem von den Serienmacherinnen individuell gewählten spezifischen Mischungsverhältnis der beiden Pole ergibt. Der Rezensent gibt dies auch unumwunden zu, indem er zugesteht, dass eine möglichst realitätsnahe Darstellung eine schlechte Dramaturgie bedingt. Konventionelle Erzählungen brauchen Protagonist^innen, die heldenhaft Missstände aufdecken, anklagen, und dadurch die Welt verbessern. Insbesondere die fiktive Wissenschaftlerin Komyuk, die mal in Chernobyl recherchiert und kurz darauf am Besprechungstisch mit Michail Gorbachow sitzt und wiederholt die Konfrontation mit hochrangigen Funktionären sucht, erweitert die Skala erheblich in Richtung Fiktionalität, ist aber zugleich ein Zugeständnis an das Diversitätsanliegen aktueller Produktionen, das in einer männerdominierten Katastrophe eine weibliche Heldin etabliert sehen möchte. CHERnobyL erweitert somit trotz oder gerade wegen der - ein hohes Maß an Authentizität suggerierenden - Ausstattung die Grenzen des Dokufiktionalen, da die Serie diesem Dokumentationsanspruch eine ausgeprägt dramatisierte Aufbereitung der historischen Ereignisse zur Seite stellt.

Zusammenfassend galt es in diesem Beitrag aufzuzeigen, dass eine enge Korrelation von Wissen und Authentizität besteht - so etwa in der detailgetreuen Ausstattung und Aufbereitung von Expertenwissen, die Objektauthentizität mit Wissenskongruenz zwischen Medium und Publikum zum zentralen Moment einer filmischen Inszenierungsstrategie wählt. Ferner korreliert die Subjektauthentizität mit einer Wissensdiskrepanz zwischen Rezipient`innen und der Diegese, insbesondere durch die emotionale Involviertheit und Identifikation mit den Figuren,

27 Gessen: „What HBO’s ,Chernobyl“ Got Right, and What It Got Terribly Wrong“.

28 Gessen: „What HBO’s ,Chernobyl' Got Right, and What It Got Terribly Wrong“. 
was wiederum einhergeht mit einer kognitiven Distanz aufgrund des Wissensvorsprungs des Publikums.

CHERNOBYL zeigt exemplarisch auf, wie in einer aktuellen historischen Serienproduktion die Produktion und medial aufbereitete Einspeisung geschichtlicher Narrative ins populärkulturelle Gedächtnis funktionieren. Hiermit einhergehend wird das, was wir mithin ,Wahrheit' nennen, ganz im Sinne des eingangs erwähnten Legasov-Zitats, als ein Produkt interessengeleiteter Manipulation - hier einer medialen Inszenierungsstrategie - figuriert. Dass diese systematische Manipulation von Wahrheit im hybriden Modus des Teil-Fiktiven dramatisiert und inszeniert wird, beweist einmal mehr das altbekannte Diktum: um über Fakten zur Wahrheit zu gelangen, muss man zu den Mitteln der Fiktion greifen. Der enorme Zuspruch und die mediale Aufmerksamkeit, die die Serie erhalten hat, ist Nicholson zufolge das Resultat des aktuell zu konstatierenden „postfaktischen Zeitalters":

Facts are simply a matter of interpretation. The obfuscation of truth is rampant and catastrophic. We may not be living under the threat of the KGB, but the menace of constant surveillance does not feel far away. The rejection of scientific knowledge, of experts, has terrible consequences. It makes sense that modern audiences would take to Chernobyl; the contemporary resonance is plain. ${ }^{29}$

Indem die Serie die Frage nach dem Verhältnis von Fakt und Fiktion zwar ambivalent darstellt und verhandelt, letztlich aber doch eine klare Botschaft entwirft, bekennt sie sich entschieden zu der Möglichkeit, dass Fakten objektiv rekonstruierbar sind und die wie auch immer geartete Wahrheit damit aufgedeckt werden kann und muss. Dass sie dies ausgerechnet im hybriden Modus des Dokufiktionalen tut, entbehrt nicht einer gewissen Ironie, zeigt jedoch die Wirkmächtigkeit von Fiktionen auf.

\section{Filmografie}

Chernobyl. Reg. Johan Renck. HBO 2019.

29 Nicholson: „Chernobyl“. 


\section{Literatur}

Fountain, Henry. „Plenty of Fantasy in HBO’s ,Chernobyl', but the Truth Is Real“. In: New York Times vom 02.06.2019. https://www.nytimes.com/2019/06/02/arts/television/ chernobyl-hbo.html (27.06.2020).

Gerhardt, Daniel. „Der Hype überstrahlt alles“. In: Zeit online vom 19.06.2019. https://www. zeit.de/kultur/film/2019-06/chernobyl-hbo-serie-craig-mazin-sky/komplettansicht (27.06.2020).

Gessen, Masha. „What HBO’s ,Chernobyl“ Got Right, and What It Got Terribly Wrong“. In: The New Yorker vom 04.06.2019. https://www.newyorker.com/news/our-columnists/whathbos-chernobyl-got-right-and-what-it-got-terribly-wrong (27.06.2020).

Gronenthal, Mariella C. Nostalgie und Sozialismus: Emotionale Erinnerung in der deutschen und polnischen Gegenwartsliteratur. Bielefeld: transcript 2018.

Hale, Mike. „,Chernobyl', the Disaster Movie“. In: New York Times vom 03.05.2019. https:// www.nytimes.com/2019/05/03/arts/television/review-chernobyl-hbo.html (27.06.2020).

Hattendorf, Manfred. Dokumentarfilm und Authentizität: Ästhetik und Pragmatik einer Gattung. Konstanz: UVK Medien 1999.

Koschorke, Albrecht. „Das Mysterium des Realen in der Moderne“. In: Auf die Wirklichkeit zeigen: Zum Problem der Evidenz in den Kulturwissenschaften. Ein Reader. Hg. Helmut Lethen, Ludwig Jäger, Albrecht Koschorke. Frankfurt a. M.: Campus Verlag 2015, 13-38.

Neef, Christian. „,Das Modell taugt nichts““. In: Der Spiegel 12 (2011), 111-112.

Nicholson, Rebecca. „Chernobyl: Horrifying, Masterly Television that Sears on to Your Brain“. In: The Guardian vom 29.05.2019. https://www.theguardian.com/tv-and-radio/ 2019/may/29/chernobyl-horrifying-masterful-television-that-sears-on-to-your-brain (27.06.2020).

Pfister, Manfred. Das Drama. München: Fink 1977.

Pirker, Eva Ulrike/Rüdiger, Mark. „Authentizitätsfiktionen in populären Geschichtskulturen: Annäherungen“. In: Echte Geschichte. Authentizitätsfiktionen in populären Geschichtskulturen. Hg. Eva Ulrike Pirker u. a. Bielefeld: transcript 2010, 11-30.

Schmidt, Siegfried J. Lernen, Wissen, Kompetenz, Kultur: Vorschläge zur Bestimmung von vier Unbekannten. Heidelberg: Auer 2005.

Wiegandt, Markus. Chronisten der Zwischenwelten: Dokufiktion als Genre. Operationalisierung eines medienwissenschaftlichen Begriffs für die Literaturwissenschaft. Heidelberg: Winter 2017.

Wolf, Werner. Ästhetische Illusion und Illusionsdurchbrechung in der Erzählkunst: Theorie und Geschichte mit Schwerpunkt auf englischem illusionsstörenden Erzählen. Tübingen: Max Niemeyer Verlag 1993. 
